\title{
ESTUDIO COMPUTACIONAL DE RMN DEL ACETAMINOFÉN Y SUS PRECURSORES QUÍMICOS
}

\section{NMR COMPUTATIONAL STUDY OF ACETAMINOPHEN AND ITS CHEMICAL PRECURSORS}

\section{Sebastián Cuesta H. ${ }^{1}$, Shirley Morocho P. ${ }^{2}$ \& Lorena Meneses O. ${ }^{2}$}

Recibido: 8 febrero 2018 / Aceptado: 26 marzo 2018

DOI: 10.26807/ia.v6i2.75

Palabras claves: Acetaminofén, espectroscopia, Gaussian03, modelación, RMN.

Keywords: Acetaminophen, Gaussian03, modeling, NMR, spectroscopy.

\section{RESUMEN}

En este artículo, se presenta un estudio espectroscópico computacional de Resonancia Magnética Nuclear del acetaminofén y sus principales precursores químicos: el 4-nitrofenol y el 4-aminofenol. Se realizó la optimización molecular de todas las estructuras de interés y, con estas estructuras, se calcularon las propiedades espectroscópicas de resonancia magnética nuclear (RMN). Todos los cálculos fueron realizados utilizando el programa de cálculo electrónico estructural GAUSSIAN 03®. Para los espectros de protón y carbono se utilizó trimetilsilano (TMS) como compuesto de referencia, para los espectros de oxígeno se utilizó como referencia agua y para los de nitrógeno, amoniaco. Los espectros computacionales de ${ }^{1} \mathrm{H}-\mathrm{RMN},{ }^{13} \mathrm{C}-\mathrm{RMN},{ }^{17} \mathrm{O}-\mathrm{RMN}$ y ${ }^{15} \mathrm{~N}-\mathrm{RMN}$ 
fueron comparados con espectros experimentales y tablas de absorción RMN de diferentes átomos y grupos funcionales obtenidos de la literatura, y se encontró que los espectros calculados presentan las mismas bandas de absorción. Las principales diferencias encontradas se deben al estado de agregación, ya que los cálculos computacionales se realizaron en estado gaseoso, sin presencia de solvente, mientras que los obtenidos de la literatura se encuentran en soluciones deuteradas. Esto demuestra que es posible realizar análisis espectroscópico de RMN mediante cálculos electrónicos computacionales, con un alto nivel de satisfacción, lo que puede ayudar en la elucidación estructural, en los casos en los que resulta imposible utilizar la técnica experimental.

\section{ABSTRACT}

In this research, a Nuclear Magnetic Resonance spectroscopic computational study of acetaminophen and its main chemical precursors: 4-nitrophenol and 4-aminophenol is presented. An optimization of all the molecular structures was performed. With the optimized structures, spectroscopic properties of nuclear magnetic resonance (NMR) were calculated. All calculations were performed using the structural electronic program GAUSSIAN 03 ${ }^{\circledR}$. For the Hydrogen and Carbon spectra, Trimethylsilane (TMS) was used as the reference compound, for the Oxygen spectra water was used as reference and for Nitrogen, ammonia was used. The computational spectra of ${ }^{1} \mathrm{H}-\mathrm{NMR},{ }^{13} \mathrm{C}-\mathrm{NMR},{ }^{17} \mathrm{O}-$ NMR and ${ }^{15} \mathrm{~N}-\mathrm{NMR}$ were compared with experimental spectra and NMR absorption tables of different atoms and functional groups obtained from the literature, showing the same absorption peaks in the calculated spectra. The main differences were due to the state of aggregation, since the computational calculations were carried out in the gas phase, without a solvent, while those obtained from the literature are obtained in deuterated solutions This shows that it is possible to perform NMR spectroscopic analysis using computational electronic calculations, with a high level of satisfaction, which can help in structural elucidation, in cases where it is impossible to use the experimental technique. 


\section{INTRODUCCIÓN}

La química computacional es una rama de la química que utiliza ordenadores para ayudar a resolver problemas químicos. Utiliza los resultados de la química teórica, incorporados en algún programa computacional, para calcular las estructuras y las propiedades de moléculas y cuerpos sólidos. Mientras sus resultados normalmente complementan la información obtenida en experimentos químicos, pueden, en algunos casos, predecir fenómenos químicos no observados a la fecha. La química computacional es ampliamente utilizada en el diseño de medicamentos y materiales (Morales, 2013).

Los estudios computacionales pueden Ilevarse a cabo con el fin de encontrar un punto de partida para la síntesis de laboratorio, ayudar en el entendimiento de datos experimentales, predecir moléculas desconocidas o explorar mecanismos de reacción que no han sido fáciles de estudiar mediante experimentos (Morales, 2013). En todos los casos, el tiempo de cómputo aumenta rápidamente a medida que el tamaño del sistema estudiado crece.
El acetaminofén o paracetamol, es uno de los medicamentos más utilizados en el mundo. Es mundialmente reconocido por su efectividad para combatir el dolor muscular y la cefalea (dolor de cabeza persistente). Es un analgésico suave cuya principal actividad farmacológica es reducir la temperatura de los pacientes con fiebre; además, posee una pequeña actividad antinflamatoria. El acetaminofén se aplica también para el dolor general, como uno de sus principales usos (Fernández et al., 2012).

El acetaminofén está considerado como el tratamiento de elección como antipirético y analgésico frente a otros AINES (antiinflamatorios no esteroideos) y en especial, frente a la aspirina, ya que no presenta muchos de los efectos adversos que estos producen, como hemorragia digestiva alta (Fernández et al., 2012). Además, tiene un perfil de seguridad adecuado por sus propiedades, en comparación con otros fármacos (El Colombiano, 2015).

En la actualidad, hay más de 90 productos que contienen acetaminofén, 
que están disponibles en el mostrador de las farmacias como medicamentos de libre venta (OTC por sus siglas en inglés). Muchos de ellos se venden como tratamientos para el alivio del resfriado, gripe y pueden ser comprados en una serie de diferentes formulaciones (Ellis, 2002).

El acetaminofén (nombre IUPAC N(4-hidroxifenil)etanamida), cuya estructura química se observa en la Figura 1, se encuentra dentro de los antinflamatorios no esteroideos debido a sus acciones analgésicas y antipiréticas, dentro del grupo de los derivados del $p$-aminofenol. El acetaminofén se sintetizó a finales de 1800 y su actividad analgésica fue reportada por Josef von Mering en 1893. Sin embargo, no fue hasta la segunda mitad del siglo XX, que llegó a ser ampliamente utilizado (Ellis, 2002; Kaplowits \& Deleve, 2013). Hoy en día, se lo puede encontrar con una infinidad de nombres que dependen de la casa farmacéutica y del país. Algunos nombres son: Analgan Parabay, acetaminofén MK, Agudol, etc. (PLM, 2011).

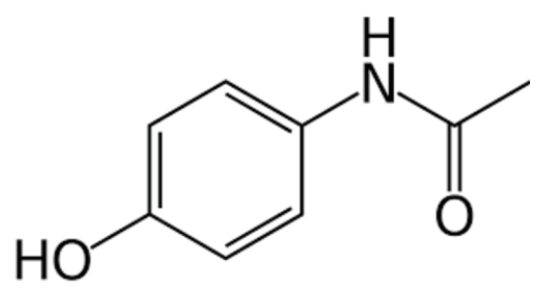

Figura 1. Estructura de acetaminofén

La Resonancia Magnética Nuclear (RMN) es la herramienta analítica que proporciona la mayor información estructural, incluyendo la estereoquímica, en un tiempo asequible. La técnica no es destructiva y tiene aplicaciones en todas las áreas de la Química. La RMN es una espectroscopía de absorción, cuyo fundamento es la absorción de energía de radiofrecuencias, por un núcleo magnéticamente activo. Este, orientado en el seno de un campo magnético, cambia su orientación al absorber energía de cierta longitud de onda (Universidad de Sevilla, 2010).

Las partes fundamentales de un espectrómetro de RMN son un imán, un oscilador de radiofrecuencias que suministra la energía necesaria para cambiar la orientación de los núcleos, una bobina detectora que recibe las señales y un sistema informatizado que gobierna todo el 
equipo y que incluye un sistema de amplificación y registro (Universidad de Sevilla, 2010).

Entre los núcleos magnéticamente activos más frecuentes en los compuestos orgánicos se encuentran: protón ${ }^{1} \mathrm{H}$, carbono ${ }^{13} \mathrm{C}$, nitrógeno ${ }^{15} \mathrm{~N}$ y fósforo ${ }^{31} \mathrm{P}$ (Universidad de Sevilla, 2010).
El propósito de este trabajo fue realizar un estudio de resonancia magnética nuclear computacionalmente, para identificar las principales bandas de absorción del acetaminofén y sus precursores de síntesis, a fin de compararlos con los espectros de la literatura y comprobar la eficiencia de la técnica computacional para la elucidación estructural.

\section{MATERIALES Y MÉTODOS}

Todos los cálculos se realizaron en el estado fundamental optimizado de las moléculas al nivel de teoría B3LYP/6-311G $(d, p)$, implementado en el paquete de programas Gaussian 03 (Frisch et al., 2003). Se utilizó el programa Gauss View 4.1 como software de visualización. Para obtener los espectros ${ }^{1} \mathrm{H}-\mathrm{RMN},{ }^{13} \mathrm{C}-\mathrm{RMN}$, ${ }^{17} \mathrm{O}-\mathrm{RMN},{ }^{15} \mathrm{~N}-\mathrm{RMN}$, se utilizó la entrada \#NMR=all dentro del modelo
GIAO (orbitales atómicos que incluyen calibración).

Para que los espectros de hidrógeno y de carbono sean comparables con los de la literatura, se utilizó TMS como compuesto de referencia. Para los espectros de oxígeno se utilizó agua como compuesto de referencia y para los espectros de nitrógeno se utilizó amoniaco.

\section{RESULTADOS}

Como se puede observar en la Figura 2 , el espectro RMN es un gráfico que registra las señales de resonancia en forma de picos. Para la interpretación de un espectro RMN, se deben tomar en cuenta cuatro aspectos: el número, la posición, la multiplicidad y el área de las señales. El número de señales presentes en un espectro corresponden al número de tipos de hi- 
drógeno, carbonos, oxígenos y nitrógenos distintos existentes en la molécula (Palop, 2009).

Se obtuvieron los espectros RMN para las especies principales de la reacción: 4-nitrofenol, 4-aminofenol y acetaminofén, como producto final. En la Figura 2 se aprecian los espectros ${ }^{1} \mathrm{H}-\mathrm{RMN}$ para estas tres moléculas, donde se pueden observar las principales bandas de absorción y los números de los átomos de hidrógeno que representan.

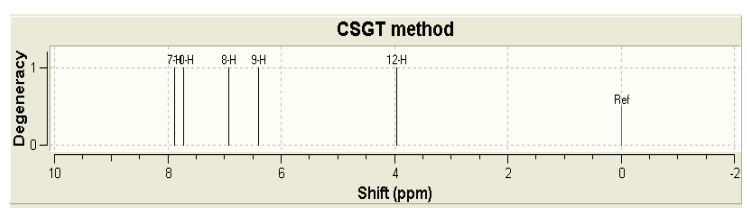

a)

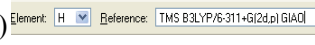

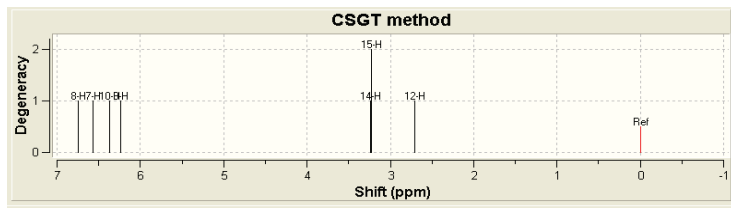

b) Element: $H \otimes$ Reference: TMS BSLYP/6/311+Gi/2dplalikd

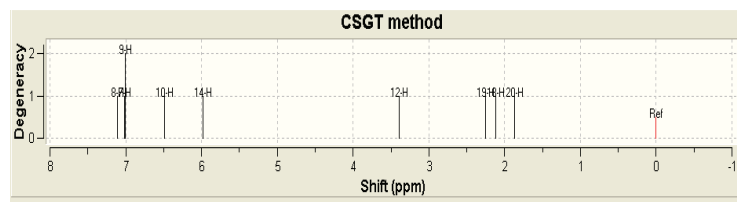

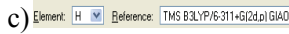
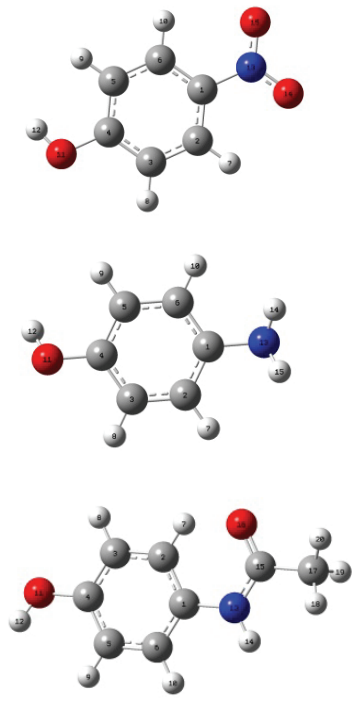

Figura 2. Espectros computacionales de 'H-RMN de las moléculas en estudio. a) 4-nitrofenol, b) 4-aminofenol, c) acetaminofén

Para el caso del 4-nitrofenol, se puede observar que aproximadamente a 4,0 ppm de desplazamiento químico aparece la banda correspondiente al hidrógeno del grupo hidroxilo (hidró- geno 12), mientras que entre $6,5 \mathrm{y}$ 8,0 ppm se observan los cuatro hidrógenos del anillo aromático (hidrógenos $7,8,9$ y 10). 
Para el caso del 4-aminofenol, se puede observar que los hidrógenos 7 , 8, 9, 10 y 12 están en las mismas posiciones que para el 4-nitrofenol, pero además, existen dos bandas adicionales a 3,3 ppm aproximadamente, correspondientes a los hidrógenos del grupo amino (hidrógenos 14 y 15).

Para el acetaminofén, se puede observar que aparecen las mismas bandas indicadas para el 4-nitrofenol, con ligeras variaciones en la posición, debido al ambiente químico, y además, tres bandas muy cercanas, alrededor de 2,0 ppm, correspondientes a los tres hidrógenos del metilo del grupo acetilo (hidrógenos 18, 19 y 20), y una banda a 6,0 ppm correspondiente al hidrógeno del grupo amido (hidrógeno 14).

En la Figura 3 se muestran los espectros computacionales de RMN de carbono $\left({ }^{13} \mathrm{C}-\mathrm{RMN}\right)$ para las tres moléculas estudiadas, donde se pueden observar las principales bandas de absorción y los números de los átomos de carbono a los que representa cada banda.

En el caso del 4-nitrofenol, se pueden observar seis bandas que aparecen en un desplazamiento que va de 115 a 165 ppm aproximadamente, correspondientes a los seis carbonos del anillo aromático. Se puede ver la equivalencia de los cuatro carbonos enlazados únicamente a hidrógenos (carbonos 2, 3, 5 y 6) en un desplazamiento de 115 a 130 ppm, mientras que el carbono enlazado al nitrógeno del grupo nitro (carbono 1) se observa a 145 ppm aproximadamente, y el enlazado al oxígeno del grupo hidroxilo (carbono 4) a 165 ppm.

Para el 4-aminofenol, las bandas aparecen de manera similar que para el 4-nitrofenol, con ligeros desplazamientos debidos al ambiente químico de la molécula. Se observan también seis bandas, pero en este caso, las bandas de los carbonos enlazados a hidrógeno están superpuestas totalmente (carbonos 2, 3, 5 y 6), alrededor de 115 ppm aproximadamente. El carbono enlazado al nitrógeno del grupo amino se observa a 145 ppm, mientras que el enlazado al oxígeno del grupo hidroxilo alrededor de 155 ppm.

En el caso del acetaminofén, el espectro presenta varias diferencias con 
respecto a los dos precursores de síntesis. Por un lado, los carbonos del anillo aromático enlazados a hidrógeno ya no son equivalentes, el carbono 6 está a 160 ppm aproximadamente, muy alejado de los carbonos 2,3 y 5, que aparecen entre 115 y 135 ppm. Luego, el carbono 1, enlazado al nitrógeno del grupo amido, se observa alrededor de 120 ppm, diferente al caso del 4-aminofenol y del 4-nitrofenol. Así mismo, el carbono 4, enlazado al oxígeno del grupo hidroxilo está a 130 ppm, muy debajo del valor observado para los precursores. En este caso, además, existen dos bandas adicionales, correspondientes a los carbonos del grupo acetilo, en 165 ppm el carbono enlazado al oxígeno (carbono 15) y en 20 ppm el carbono del grupo metilo (carbono 17).

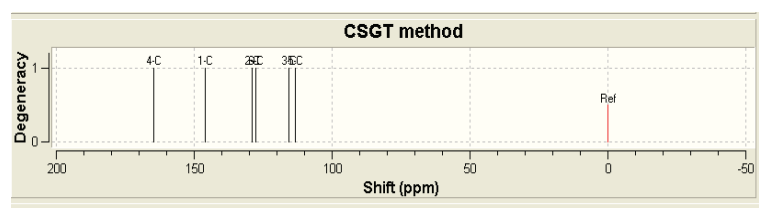

a)
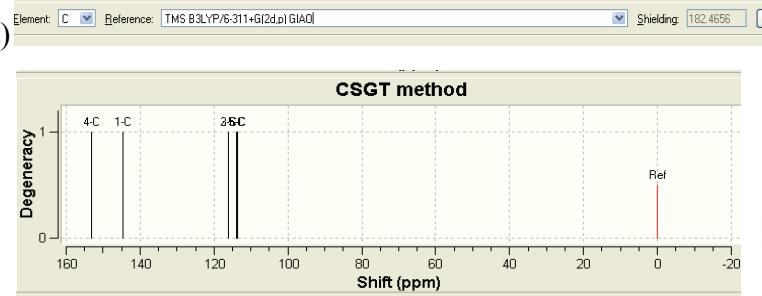

b)

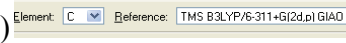
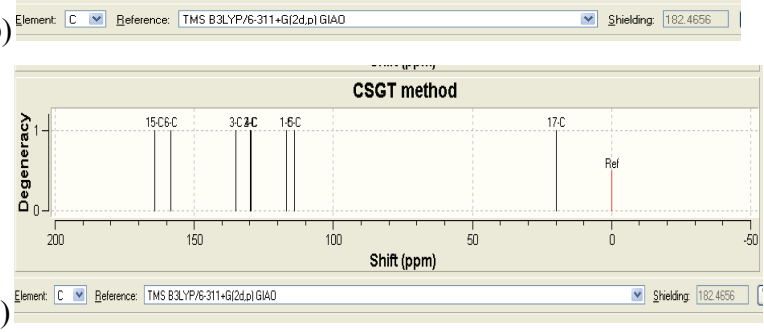
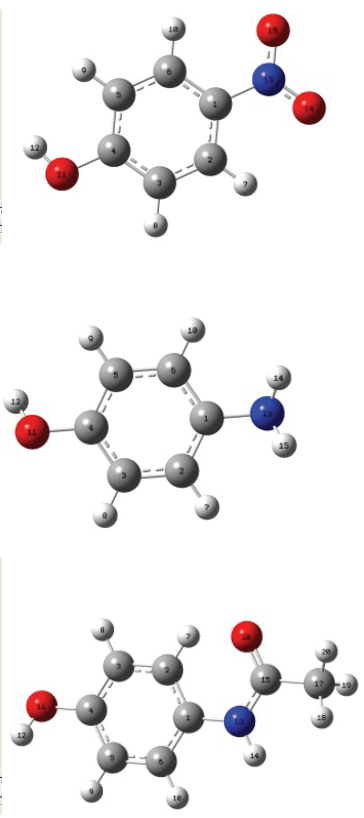

Figura 3. Espectros computacionales de ${ }^{13} \mathrm{C}-\mathrm{RMN}$ de las moléculas estudiadas. a) 4-nitrofenol, b) 4-aminofenol, c) acetaminofén 
Computacionalmente, el programa permite obtener el espectro de RMN del ${ }^{17} \mathrm{O}$ y de ${ }^{15} \mathrm{~N}$. Estos espectros se presentan en las Figuras 4 y 5 . Se pueden observar los picos de absorción y la numeración de los átomos a los que corresponde cada banda.

En la Figura 4 se puede observar que aparecen tres bandas para el caso del 4-nitrofenol, correspondientes al oxígeno del grupo hidroxilo a 150 ppm (oxígeno 11) y a los dos oxígenos del grupo nitro alrededor de 640 ppm (oxígenos 14 y 15). Para el caso del 4-aminofenol aparece una sola banda alrededor de 125 ppm correspondiente al oxígeno del grupo hidroxilo. En el espectro del acetaminofén se pueden observar dos bandas, una correspondiente al oxígeno del grupo hidroxilo aproximadamente a 140 ppm, y otra correspondiente al oxígeno del grupo acetilo alrededor de 440 ppm.

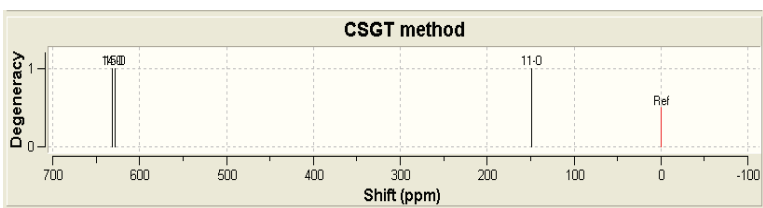

a)

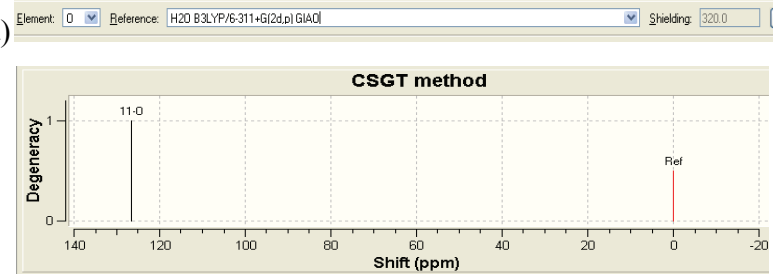

b)

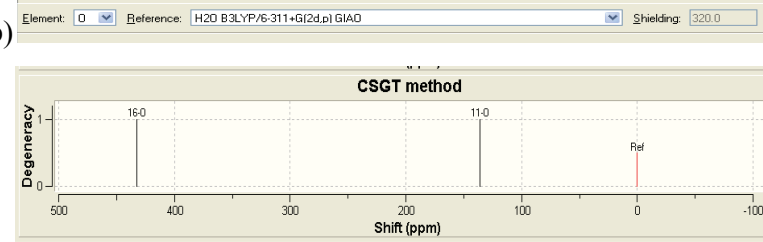

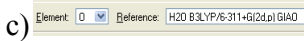
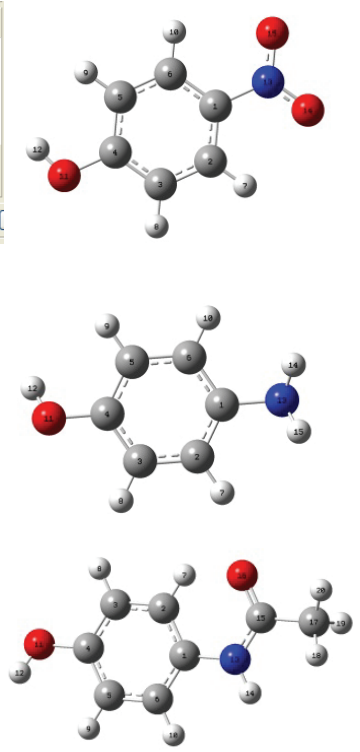

Figura 4. Espectros computacionales de ${ }^{17} \mathrm{O}-\mathrm{RMN}$ de las moléculas estudiadas. a) 4-nitrofenol, b) 4-aminofenol, c) acetaminofén 
En la Figura 5 se pueden observar los tres espectros de ${ }^{15} \mathrm{~N}-\mathrm{RMN}$. En cada caso, se puede apreciar solo una banda de absorción, ya que cada molécula contiene únicamente un nitrógeno en su estructura. En el 4-nitrofenol, el nitrógeno corresponde al del grupo nitro, y aparece a 395 ppm aproximadamente. En el caso del 4aminofenol, el nitrógeno corresponde al grupo amino y aparece a 85 ppm. En el acetaminofén, el nitrógeno corresponde al grupo amido, y aparece aproximadamente a 150 ppm.

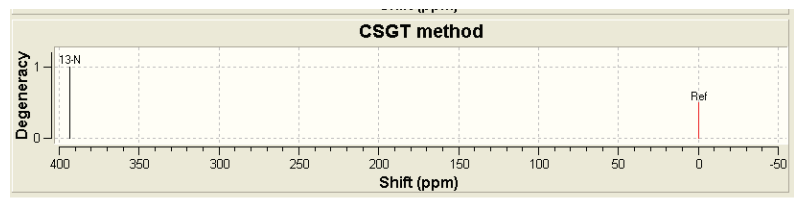

a)

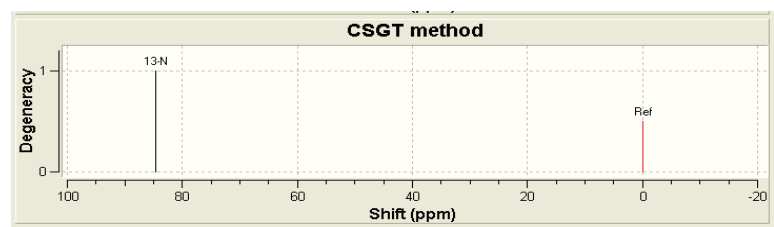

b)

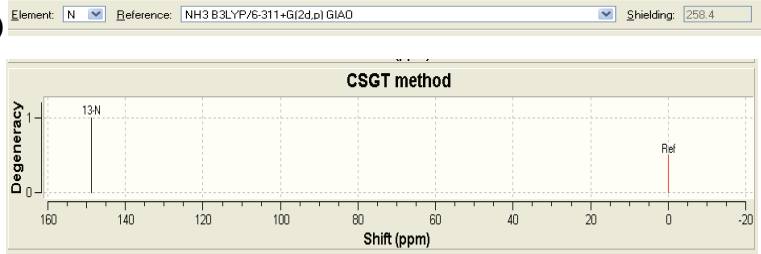

c)

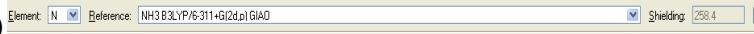

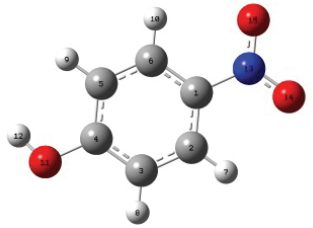
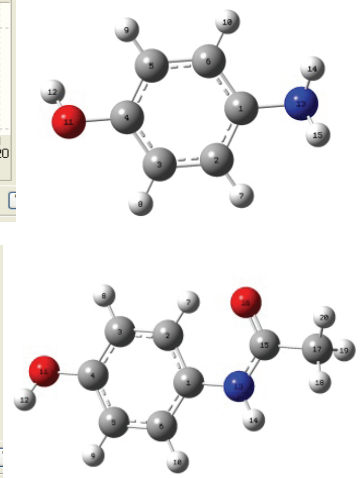

Figura 5. Espectros computacionales de ${ }^{15} \mathrm{~N}-\mathrm{RMN}$ de las moléculas estudiadas. a) 4-nitrofenol, b) 4-aminofenol, c) acetaminofén 


\section{DISCUSIÓN}

En los espectros de RMN obtenidos computacionalmente, se puede observar claramente e identificar los distintos tipos de átomos en una molécula orgánica. El programa distingue los hidrógenos alifáticos de los aromáticos, los de hidroxilo de los del grupo amino, y con mayor detalle, los hidrógenos del grupo amino de los del grupo amido. Cada hidrógeno aparece a un desplazamiento químico distinto, considerando todo el ambiente químico de la molécula.

También se diferencian los distintos tipos de carbono, oxígeno y nitrógeno, en función del grupo funcional al que pertenecen. En los espectros, el número de señales presentes corresponde con el número de átomos distintos existentes en la molécula. En la Figura 2, el acetaminofén presenta nueve hidrógenos en su molécula que se pueden ver en el espectro. Los hidrógenos 10, 12 y 14 se presentan como singletes, es decir señales compuestas de un único pico. También, es frecuente encontrar espectros con señales múltiples conteniendo varios picos con una proporción definida, lo que se conoce como acoplamiento spin-spin, esto se puede apreciar en los hidrógenos 9 y 7 correspondientes al anillo bencénico, y en los hidrógenos 18 y 17 correspondientes al $-\mathrm{CH}_{3}$ del grupo acetilo. Este efecto de acoplamiento spin-spin solo es visible entre hidrógenos de carbonos vecinos (Palop, 2009).

El espectro ${ }^{1} \mathrm{H}-\mathrm{RMN}$ del acetaminofén obtenido computacionalmente fue comparado con el espectro experimental. En la Figura 6 se muestra el espectro ${ }^{1} \mathrm{H}-\mathrm{RMN}$ experimental del acetaminofén obtenido de la literatura (Universidad Nacional de Colombia, s.f.).

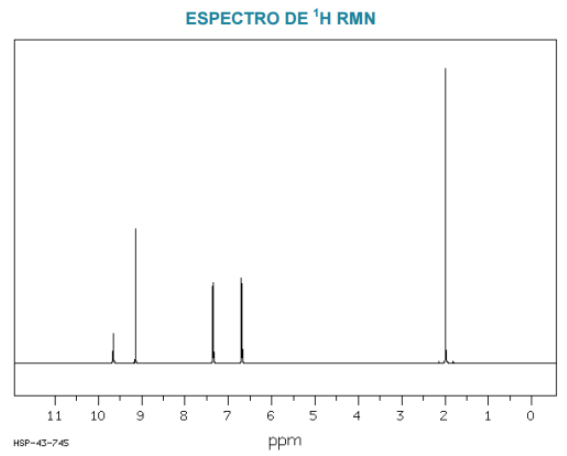

Figura 6. Espectro experimental de 'H-RMN de acetaminofén (Universidad Nacional de Colombia, s.f.) 
Al comparar ambos espectros se pueden observar varias diferencias. La principal se encuentra en el número de picos que poseen los espectros; mientras el espectro experimental posee cinco picos, el obtenido computacionalmente presenta ocho. En el espectro experimental, se puede evidenciar que el pico obtenido a los 2 ppm contiene una señal múltiple, lo mismo que el pico a los 6,5 ppm y el pico a los 7,5 ppm. Estas señales múltiples indican que la señal producida por varios hidrógenos está superpuesta, ya que son químicamente iguales. En el espectro computacional, ya que se basa en un cálculo matemático, se pueden identificar con mayor detalle pequeñas diferencias en el ambiente químico, mostrando diferentes picos en los casos en los que en el espectro experimental se superponen.

Esto se puede observar en el grupo metilo. A simple vista, se podría decir que los tres hidrógenos del grupo metilo tienen el mismo ambiente químico, por lo tanto es correcto que produzcan una sola señal a los 2 ppm. Al observar más detenidamente, el ambiente químico del hidrógeno 20 es ligeramente diferente a los otros dos, ya que tiene influencia del oxígeno 16. Lo mismo pasa con el hidrógeno 18, que podría estar influenciado ligeramente por el hidrógeno 14 y el nitrógeno 13. Lo mismo sucede con los hidrógenos que se encuentran en el grupo aromático.

En los espectros representados en la Figura 3, cada tipo de carbono aparece con un pico a un ppm específico. El acetaminofén presenta 8 carbonos en su molécula los cuales son claramente observables en el espectro. Con este tipo de átomo, las señales también pueden ser singletes como las observadas para el carbono $17,15,3$ y 6 . De igual manera, los átomos de carbono presentan acoplamientos spin-spin como se ve en los carbonos 2-4 y 5-6 correspondientes al anillo bencénico.

En la Figura 7 se muestra el espectro experimental de ${ }^{13} \mathrm{C}-\mathrm{RMN}$ obtenido de la literatura (Universidad Nacional de Colombia, s.f.).

En el espectro experimental se pueden apreciar seis señales, mientras que en el espectro computacional aparecen siete, esto se debe a que 
igual que en el caso del espectro de hidrógeno, existen señales superpuestas. Además, se puede ver que los picos en el espectro computacional están recorridos hacia un mayor desplazamiento en comparación al experimental, lo cual podría deberse principalmente a la diferencia en el estado de agregación, ya que los cálculos computacionales se los hicieron en estado gaseoso y el experimental se realizó en solución.

ESPECTRO DE ${ }^{13} \mathrm{C}$ RMN

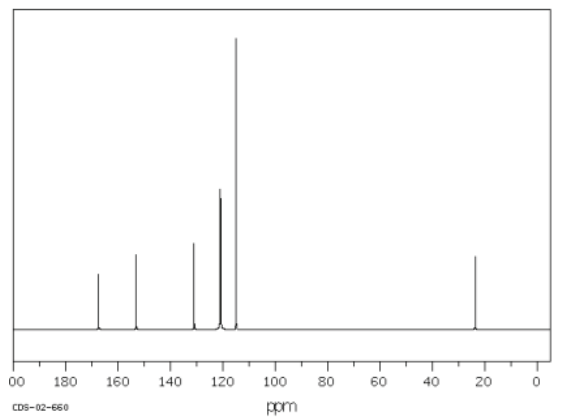

Figura 7. Espectro experimental de ${ }^{13} \mathrm{C}$-RMN del acetaminofén (Universidad Nacional de Colombia, s.f.)

A los 120 ppm aparece una señal múltiple en el espectro experimental, en cambio en el computacional aparece a los 130 ppm, correspondiente a los carbonos del anillo bencénico. Sin embargo, ambos espectros son comparables notándose el mismo patrón; un primer pico a 20 ppm correspondiente al carbono 17 del grupo metilo (en el espectro experimental aparece alrededor de los 24 ppm), luego no existe ningún pico hasta pasado los 100 ppm donde se muestra el resto de señales hasta antes de los 170 ppm.

En los espectros de la Figura 4, el número de señales corresponde al número de oxígenos presentes en la molécula con un ambiente químico diferente. Así, en el espectro del 4-nitrofenol, los oxígenos 14 y 15 presentan el mismo ambiente químico. En el caso de tener un espectro de ${ }^{17} \mathrm{O}-\mathrm{RMN}$ experimental de esta molécula, estos oxígenos se presentarían como una sola señal. En el caso del espectro computacional, la señal está casi superpuesta. El espectro del 4-aminofenol presenta una sola banda, debido a que presenta solo un oxígeno en su estructura. El acetaminofén presenta dos oxígenos en su molécula que se encuentran en dos ambientes totalmente diferentes, uno perteneciente al oxígeno del fenol y el otro al del grupo acetil, por lo que aparecen dos señales singletes en el espectro. En los tres casos, el oxígeno 
del grupo hidroxilo en el anillo aromático en la posición para debe aparecer entre los 126 y 146 ppm (Prestch y Badertscher, 2009). En el espectro computacional de ${ }^{17} \mathrm{O}-\mathrm{RMN}$ del 4-nitrofenol, 4-aminofenol y el acetaminofén, estos oxígenos aparecen entre 135-145 ppm, por lo que los espectros computacionales son totalmente comparables con los experimentales.

En los espectros ${ }^{15} \mathrm{~N}$-RMN de la Figura 5 , se puede observar que cada espectro muestra una sola señal, debido a que en todas las estructuras existe solo un átomo de nitrógeno. Los tres picos aparecen en lugares totalmente diferentes de los espectros, debido a que el ambiente químico circundante es diferente en los tres casos. El 4-nitrofenol posee un singlete debido al grupo $-\mathrm{NO}_{2}$, el 4-ami- nofenol uno del grupo $-\mathrm{NH}_{2}$, y el acetaminofén una banda correspondiente al nitrógeno del enlace amida (N-C).

La literatura indica que el rango para la señal del grupo amina es entre 40 y 90 ppm (Prestch y Badertscher, 2009), en el espectro computacional obtenido para el 4-aminofenol, la señal se observa a los 85 ppm, por lo que se encuentra dentro del rango aceptado. Para el nitrógeno unido con el grupo acetil, la literatura muestra un rango entre 150-170 ppm (Prestch y Badertscher, 2009). En el espectro computacional está en 150 ppm. En el grupo nitro, el nitrógeno debe aparecer en los 355-395 ppm (Instituto de Química, 2016), en el espectro computacional, se obtuvo una señal a los 395 ppm.

\section{CONCLUSIONES}

En este estudio, se logró demostrar que los métodos computacionales para la obtención de espectros de RMN de diferentes átomos son totalmente comparables con resultados obtenidos experimentalmente, logrando ser bastante precisos. Las pequeñas diferencias encontradas al comparar espectros experimentales con los obtenidos computacionalmente, se deben principalmente al estado de agregación de la molécula, ya que en el estudio computacional se utilizó la molécula en estado gaseoso, mientras que en el análisis experimental, las moléculas se encuentran disueltas en agua deuterada. 


\section{LISTA DE REFERENCIAS}

El Colombiano. (2015), Conozca más sobre el acetaminofén, http://www.elcolombiano .com/colombia/salud/conozca-mas-sobre-el-acetaminofen-DX2572410

Ellis, F. (2002). Paracetamol- A curriculum resource, London: Royal Society of Chemistry, British library.

Fernández, P.L., Moreno, A., Leza, J.C., Lizasoain, I., Moro, M.A., Portolés, A. (2012). Velázquez. Manual de farmacología básica y clínica (18. ed.) Buenos Aires: Editorial Médica Panamericana.

Frisch, M.J., Trucks, G.W., Schlegel, H.B., Scuseria, G.E., Robb, M.A., Cheeseman, J.R., Scalmani, G., Barone, V., et. al. (2003). Wallingford CT: Gaussian, Inc.

Instituto de Química. (2016). http://chem.ch.huji.ac.il/nmr/techniques/1d/row2/o.html \#top

Kaplowitz, N. y Deleve, L. (2013). Drug induce liver disease (3a. ed.). New York: Elservier.

Morales, M. (2013). Química Computacional, http://plumaslibres.com.mx/2013/11/07/ quimica-computacional

Palop, J. (2009). Tutorial para la interpretación de espectros de resonancia magnética nuclear del protón http://webcache.googleusercontent.com/search?q=cache:iyLLgNFO0h4J:www.unav.es/ocw/avanzada_q/TUTOR_RMN.pdf+\&cd=1\&hl=es$419 \& \mathrm{ct}=\mathrm{c} \operatorname{lnk} \& g \mathrm{I}=\mathrm{ec}$

PLM. (2011). Farmaprecios guía de precios sugeridos al público, promedio del mercado de y para farmacias independientes. Farma, 51, 18-26.

Prestch, E. y Badertscher, M. (2009). Structure Determination of Organic Compounds (4a. ed.). Switzerland: Springer. 
infoANALÍTICA 6-2

Julio 2018

Universidad Nacional de Colombia. (s.f.) Espectroscopia del Acetaminofén, http://168. 176.60.11/cursos/ciencias/2015657/u6/pdf/anexo_4.pdf

Universidad de Sevilla. (2010). Resonancia Magnética Nuclear, https://investigacion .us.es/scisi/sgi/servicios/rmn 